\title{
Métodos para estimar requerimientos de cal en suelos ácidos acrisoles ferricos en Xalapa, Veracruz, México
}

Methods for estimating lime requirements in acidic ferric acid soils in Xalapa, Veracruz, Mexico

Castillo Rocha Doris. G. ${ }^{1 凶}$, Segura Castilla Ana C. ${ }^{1}$, Martínez Hernández María de J. ${ }^{1}$, Lara Capistrán L. ${ }^{1}$ y Aguirre López E. ${ }^{1}$

${ }^{1}$ Universidad Veracruzana, Facultad de Ciencias Biológicas y Agropecuarias.

${ }^{凶}$ Autor para correspondencia: dorisgcr@gmail.com

Recibido: 15/04/2019

Aceptado: 15/05/2019

\section{RESUMEN}

Por su ubicación geográfica, México posee regiones tropicales y subtropicales, dichas regiones están limitadas por la marcada acidez de los suelos, siendo este fenómeno uno de los problemas más importantes en la producción de algunos cultivos. La práctica que se utiliza con el propósito de neutralizar la acidez y corregir los problemas que ésta ocasiona es el encalado de los suelos, sin embargo, para realizar esta práctica, debe considerarse la cantidad de cal por aplicar. La necesidad de cal varía de un suelo a otro, dependiendo de su naturaleza o del cultivo de que se trate, por lo que esta se debe determinar pare cada uno en particular. El suelo utilizado en esta investigación se localiza en las fincas cafetaleras “Las Animas 1' y “El Progreso", en el municipio de Xalapa, Ver. Los resultados de este trabajo muestran que los métodos Houba y Van Schouwenburg, Dunn y SMP-Simple permiten estimar RC, para los pH considerados, con mayor exactitud que los métodos de Keeney y Corey (1963), Adams y Evans (1962), Acidez hidrolítica o de Kappen modificado (1976), Acidez intercambiable (1970) y el de Morejon (1988).

Palabras clave: Acidez, Producción, Cultivos, Productividad.

\begin{abstract}
Por su ubicación geográfica, México posee regiones tropicales y subtropicales, diversas regiones están limitadas por la marcada acidez de los suelos, siendo este fenómeno uno de los problemas más importantes en la producción de algunos cultivos. La práctica que se utiliza con el propósito de neutralizar la acidez y corregir los problemas que esta ocasiona es el encalado de los suelos, sin embargo, para realizar esta práctica, debe limitar la cantidad de cal por aplicación. La necesidad de cal cambia de un suelo a otro, modifica de su naturaleza o del cultivo de que setrate, por lo que esta se debe determinar
\end{abstract}


cada uno en particular. El suelo utilizado en esta investigación se localiza en las fincas cafetaleras "Las Ánimas1" y "El Progreso", en el municipio de Xalapa, Ver. Houba y Van Schouwenburg, Dunn y SMPSimple permitir estimar RC, para los pH determinados, con mayor precisión que los métodos de Keeney y Corey (1963), Adams y Evans (1962), Acidez hidrolítica o de Kappen modificado (1976), Acidez intercambiable (1970) y el de Morejón (1988).

Keywords: Acidez, Producción, Cultivos, Productividad.

\section{INTRODUCCIÓN}

México es uno de los países con mayor diversidad en el mundo, por su ubicación geográfica posee regiones tropicales y subtropicales, dichas regiones están limitadas por la marcada acidez de los suelos, siendo este fenómeno uno de los problemas más importantes en la producción de algunos cultivos. La práctica que se utiliza con el propósito de neutralizar la acidez y corregir los problemas que ésta ocasiona es el encalado de los suelos, sin embargo, para realizar esta práctica, debe considerarse la cantidad de cal por aplicar. La necesidad de cal varía de un suelo a otro, dependiendo de su naturaleza o del cultivo de que se trate, por lo que esta se debe determinar pare cada uno en particular.

La variedad de métodos para analizar las diferentes variables son muchos y muy diferentes; en función de estos y de la variabilidad de los suelos, los resultados que se obtienen con cada uno de ellos son divergentes, por lo que es necesario determinar el más indicado por su eficacia y precisión, ya que la aplicación generalizada en todos los suelos conduce a errores en la predicción de enmiendas a aplicar.

Los diversos métodos con que se cuenta para estimar la cantidad de cal necesaria para elevar el pH del suelo a un valor deseado, son algunos bastante precisos, como el de incubación, sin embargo, requiere de mucho tiempo para su realizaban, lo cual limita su uso en análisis de rutina. Otros, que utilizan soluciones amortiguadores, permiten estimar el requerimiento de cal de manera rápida, aunque presentan el inconveniente de que no son adecuados para todos los tipos de suelos, por lo que, pare su uso, deben calibrarse. Algunos usan la titulación del suelo con una base o equilibrio con cal, los resultados que se obtienen en un tiempo razonable son casi siempre adecuados, pero también deben calibrarse pare cada tipo de suelo. Se ha sugerido que la cantidad de aluminio que se extrae con una sal neutra no tamponada puede ser empleada como índice de los requerimientos de cal, particularmente para los oxisoles y ultisoles, los suelos donde este procedimiento función, generalmente posee una capacidad de intercambio catiónica baja y una proporción de aluminio intercambiable superior a la de las bases intercambiables. Existen otros métodos que varían en su fundamento, y que algunos de ellos serán tornados en cuenta en este trabajo.

El presente estudio se realizó con el fin de comparar algunos métodos para estimar el requerimiento de cal (RC) en suelos de Xalapa, Ver, México, de acuerdo a lo antes mencionado, queda la necesidad de calibrar y ajustar métodos en relación con las circunstancias que presentaron los suelos de los sitios de estudio, por lo que, la evaluación de los métodos seleccionados se hizo mediante 
comparación de las cantidades de cal obtenidas en cada uno de ellas para circunstancias específicas y las estimadas a través de la incubación del suelo con dosis crecientes de cal.

\section{MATERIALES Y MÉTODOS}

El suelo utilizado en esta investigación se localiza en las fincas cafetaleras "Las Animas1' y "El Progreso", en el municipio de Xalapa, Ver., México, que se ubica en la parte central oeste del Estado de Veracruz, en las faldas del cerro de Macuiltepetl y las estribaciones orientales del Cofre de Perote, en la zona de transición entre la Sierra Madre Oriental y la planicie costera del Golfo de México, entre los paralelos $19^{\circ} 31^{\prime} 35^{\prime}$ 'de latitud norte y $96^{\circ} 54^{\prime}$ 51 " de longitud oeste. Tiene una altitud de 1427 msnm, colindando con los municipios de Banderilla, Coatepec, Emiliano Zapata, San Andres Tlalnelhuayocan, Naolinco y Jilotepec, (INEGI, 1993).

De acuerdo con el mapa de suelos de INEGI a escala 1:250 000 y a la clasificación FAO-UNESCO (1988), el suelo predominante es Acrisoi ferrico. Este suelo es cultivado con café, plátano, maíz y frijol, entre otros, sustentándose también bosques y pastizales. Su textura es de media a fina. Presenta una capa superficial de color ciara, pobre en materia orgánica, por lo general ácida y pobre en nutrientes y además posee acumulación de arcilla en el subsuelo (Horizonte B argico) el cual está sumamente lavado. La fertilidad de este tipo de suelo es variable según su grado de pendiente, así como su profundidad. Con frecuencia es pobre en nitrógeno, fosforo $\mathrm{y}$ potasio por, lo que continuamente es fertilizado.

En las fincas cafetaleras anteriormente mencionadas se colectaron muestras de suelo en un cuadrado de $2 \times 2 \mathrm{~m}$ se tomaron 5 muestras de la capa 0-20 $\mathrm{cm}$ de los dos puntos seleccionados, teniendo- en cuenta que estos no se localizaran en zonas de acumulación. En la figura 8 se puede apreciar un perfil del suelo Acrisol ferrico (Ultisol) de esta zona de estudio.

Algunos de los análisis fueron $\mathrm{pH}$ en agua y en cloruro de potasio (rel. 1:2); acidez hidrolítica e intercambiable; Aluminio e hidrogeno intercambiables; Cationes intercambiables (calcio, magnesio, potasio y sodio); Capacidad de intercambio catiónico y efectiva; Porcentaje de saturación por bases, por calcio, por aluminio, por materia orgánica y de nitrógeno total; Fosforo asimilable; Densidad aparente; Porcentaje de arcilla, de limo y arena; Capacidad máxima de retención de agua.

Para el procesamiento estadístico de los datos obtenidos en cada uno de los experimentos se utilizó el paquete MSTAT, elaborando el análisis de varianza y la prueba de comparación de medias de Tukey con un a de 0.01, y una vez confirmada la estimación de diferencias altamente significativas se procedió al estudio de regresión con los modelos matemáticos de mejor ajuste, las variables en estudio fueron $\mathrm{pH}$, acidez y aluminio intercambiables, calcio intercambiable, capacidad de intercambio catiónico y porcentaje de saturación de aluminio. 


\section{RESULTADOS Y DISCUSIÓN}

Cuadro 1. Propiedades físicas y químicas del suelo muestreado en "Las Animas'5 y "El Progreso".

\begin{tabular}{|c|c|c|c|}
\hline Determinación & "Las Animas" & "El Progreso" & Metodologia \\
\hline pHen agua y rel. 12 & 4.25 & 4.07 & Potenciometrico \\
\hline $\mathrm{pH}$ en KCl $1 \mathrm{~N}$ rel. $1: 2$ & 3.85 & 3.77 & Potenciometrico \\
\hline $\begin{array}{l}\text { Acider hidralitica } \\
\text { cmol kg }\end{array}$ & 3.78 & 410 & Kappen modificado \\
\hline $\begin{array}{l}\text { Acidez intercambiabie. } \\
\text { cmol } \mathrm{kg}^{-1}\end{array}$ & 2.60 & 3.20 & Kamprath \\
\hline $\begin{array}{l}\text { Aluminio intersambiable } \\
\text { cmol } \mathrm{kg}^{-1}\end{array}$ & 2.13 & 261 & Gojberg y Aguilar \\
\hline $\begin{array}{l}\text { Hidrogeno intercambiable } \\
\text { Cmol kg" }\end{array}$ & 0.67 & 0.59 & Goijberg y Aguilar \\
\hline $\begin{array}{l}\text { Calcio intercambiable } \\
\text { cmol kg }\end{array}$ & 2.16 & 3.18 & Dihel et al. \\
\hline $\begin{array}{l}\text { Magnesio intercambiable } \\
\text { cmol kg }\end{array}$ & 1.02 & 2.12 & Donel ef al. \\
\hline $\begin{array}{l}\text { Potasio intercambiable } \\
\text { cmol kg." }\end{array}$ & 0.36 & 0.12 & DTPA \\
\hline $\begin{array}{l}\text { Capacidad de intercambio } \\
\text { catiodnico cmol kg }\end{array}$ & 122 & 13.7 & Cottenie \\
\hline $\begin{array}{l}\text { Capacidad de intercambio } \\
\text { cationico efectiva, omol kg }\end{array}$ & 6.44 & 8.72 & Matemática \\
\hline $\begin{array}{l}\text { Porcentaje de saturación por } \\
\text { bases. }\end{array}$ & 29.84 & 40.30 & Matemática \\
\hline $\begin{array}{l}\text { Porcentaje de saturación por } \\
\text { calcio. }\end{array}$ & 3354 & 36.47 & Mafemática \\
\hline $\begin{array}{l}\text { Porcentaje de saturación por } \\
\text { aluminio }\end{array}$ & 33.07 & 29.93 & Matemática \\
\hline $\begin{array}{l}\text { Porcentaje de materia } \\
\text { organica. }\end{array}$ & 2.67 & 384 & Wakley-Biack \\
\hline Fósforo asimilable. ppm & 20.83 & Inapreciable & Bray \\
\hline Densidad aparente $g \mathrm{~mL}$ & 1.10 & 1. 18 & Parafina \\
\hline Porcentaje de arcilla. & 26.25 & 35.00 & Bouyoucus-Day \\
\hline Porcentaje de limo. & 2375 & 33.75 & Bouyoucus-Day \\
\hline Porcentaje de arena. & 50.00 & 31.25 & Bouyoucus-Day \\
\hline $\begin{array}{l}\text { Capacidad máxima de } \\
\text { retención de agua mL kg }\end{array}$ & 222 & 264 & Gravimitrico \\
\hline
\end{tabular}

Los valores de $\mathrm{pH}$ encontrados implican la posibilidad de la presencia de niveles altos de aluminio intercambiable, (Cajuste, 1977). Los resultados obtenidos para los sitios muestreados sobre la acidez hidrolítica fueron para el suelo de "Las Animas" de 3.78 y $4.10 \mathrm{cmol} \mathrm{kg}^{-1}$ para el suelo de "El Progreso", y es en este tipo de acidez donde se Incluye la parte menos móvil de los iones hidrogeno adsorbidos, que con mayor dificultad se intercambian por los cationes de la solución del suelo. Siendo este parámetro utilizado como un índice para determinar los RC de un sitio, (Morejon, 1987). La alta presencia de aluminio (superiores a $0.1 \mathrm{cmoi} \mathrm{kg}^{-1}$ ) puede presentar un riesgo de toxicidad para algunos cultivos.

El contenido de bases intercambiables en los sitios en estudio y de acuerdo a la clasificación de los cationes intercambiables determinados en acetato de amonio a pH 7.0 de Etchevers et al. (1971), el contenido de calcio en el suelo de "Las Animas" y "El Progreso" son 2.16 y $3.18 \mathrm{cmol}$ $\mathrm{kg}^{-1}$ respectivamente clasificándose en ambos casos como bajo. El contenido de magnesio en ambos sitios es bajo con 1.02 y 2.12 cmoles kgpara "Las Animas" y "El Progreso" respectivamente, lo mismo sucede con el potasio intercambiable. Estos bajos niveles de cationes intercambiables son provocados por el lavado de los mismos, originados por fas condiciones de la zona en su alta precipitación pluvial, extracción de bases por las plantas y por la baja liberación de ellas por los minerales primarios del suelo. Entre los cationes intercambiables y los que se encuentran en la solución hay un equilibrio químico, de tal forma que al ser absorbidos los cationes en solución por las plantas, aunado al lavado de bases, el equilibrio se restablece 
liberándose de los sitios de intercambio a la solución del suelo los cationes adsorbidos. Como la extracción y lavado de bases es continua, los suelos sufren una pérdida de ellas y los sitios de intercambio son ocupados por hidrogeno y/o aluminio, presentándose niveles tóxicos de estos elementos para la mayoría de los cultivos. La capacidad de intercambio catiónico para el suelo de "Las Animas" fue de 12.20 cmoles $\mathrm{kg}^{-1}$ de suelo, y para "El Progreso de $13.70 \mathrm{cmoles} \mathrm{kg}^{-1}$, los cuales son bajos, según el criterio de Cottenie (1980). Para la capacidad de intercambio catiónico efectiva (CICE) determinada por la suma de bases y acidez intercambiable, se obtuvieron los valores de 6.44 y 8.72 cmoles $\mathrm{kg}^{-1}$ respectivamente. Esta diferencia se debe a que la determinación de CIC se hace con acetato de amonio a $\mathrm{pH} 7.0$, por lo que a este valor de $\mathrm{pH}$ se generan cargas negativas provenientes de la materia orgánica y contenido de arcilla del suelo, sobreestimándose la CIC. Por lo que es necesario para suelos ácidos hacer una estimación correcta utilizándose la CICE. Con respecto a los valores de CICE de los sitios muestreados, 6.44 y 8.72 cmoles $\mathrm{kg}^{-1}$, resultan bajos $\mathrm{y}$, son consecuencia directa de (contenido de materia orgánica y de bases intercambiables, por lo que, a mayor contenido de materia orgánica y de bases intercambiables es mayor la CICE. En consecuencia, los suelos con una mayor CICE requieren mayor cantidad de material encalante que aquellos que presentan un valor menor. El porcentaje de saturación de bases fue para "Las Animas" de $29.84 \%$ y $40.30 \%$ para "El Progreso" y de acuerdo a la clasificación de Cottenie, (1980), el primero se dice que es bajo y medio para el segundo. Debe hacerse notar que como el porcentaje de saturación de bases, es el valor de la capacidad de intercambio cubierto por cationes distintos de los iones ácidos $(\mathrm{H}+\mathrm{y}$ A f3), depende de la CIC. El porcentaje de saturación de calcio fue bajo, y se explica por la cantidad deficiente de calcio en el complejo de intercambio catiónico del suelo de los sitios estudiados, y se espera un incremento de este porcentaje a través del encalado. El porcentaje de saturación de aluminio para los sitios estudiados de "Las Animas" y "El Progreso" fueron 30.07 y 29.93, y estos están en un nivel de toxicidad que pueden ser afectados cultivos sensibles. Elio es consecuencia de la acidez del suelo tan marcada, a los bajos niveles de cationes intercambiables y de nitrógeno.

El contenido de materia orgánica en los suelos estudiados es bajo de acuerdo a la clasificación de Fassbender y Bornemiza, (1987). Siendo 2.87 y 3.84\% para el suelo de "Las Animas" y "El Progreso" respectivamente. Es conocida la capacidad de amortiguamiento de la materia orgánica del suelo contra los cambios bruscos de pH. Esta propiedad es originada por grupos reactivos de carboxilos, fenolicos y amidicos que son capaces de protonizarse $\mathrm{O}$ desprotonizarse (ligar o liberar grupos $\mathrm{H}+$ ). Estos grupos saturados con iones hidrogeno se comportan como ácidos débiles y el hidrogeno tiene la capacidad de disociarse, generando sitios de adsorción. Es por ello que un suelo con un contenido mayor de materia organica como lo es el suelo de la finca "El Progreso" (3.84\%), los cationes ácidos (aluminio e hidrogeno) tienen una mayor tendencia a acumularse en los sitios de intercambio, requiriendo mayor cantidad de cal para elevar su $\mathrm{pH}$ a un nivel deseado desde su valor inicial, que el suelo de la finca "Las Animas" (2.87\%) con un contenido menor de materia orgánica. El contenido de nitrógeno total se encuentra directamente relacionado con el contenido de materia orgánica del suelo. Si un suelo es rico en materia orgánica lo es en nitrógeno y viceversa., tomando como referencia la clasificación de nitrógeno total de Moreno 
(1978), el suelo de "Las Animas" con $0.144 \%$ es medio; el suelo "El Progreso" con un contenido de $0.20 \%$ de nitrógeno total está clasificado como medianamente rico. Este elemento puede estar presente en el suelo en forma inorgánica, que incluye principalmente a nitratos, nitritos, amonio intercambiable y amonio fijado; y en forma orgánica está presente en la fracción húmica, principalmente en los grupos amino y en los residuos orgánicos en procesos de descomposición.

De acuerdo a la precipitación en la zona de $1504.1 \mathrm{~mm}$ (Soto y Gómez, 1993), a la textura del suelo y al bajo contenido de bases intercambiables en el contenido de nitrógeno total es de forma orgánica, originado por la lixiviación del nitrógeno inorgánico, así como la constate absorción del mismo por las plantas. El fosforo determinado por el método Bray P1 fue de 20.83 ppm para el suelo "Las Animas" e inapreciable para "El Progreso", de acuerdo con la clasificación de CSTPA (1980), se considera bajo, lo cual coincide con los datos publicados por Rossignoll (1988) para esta zona. Una de las razones comúnmente dadas para justificar el encalado de los suelos ácidos es aumentar la disponibilidad de fosforo, aunque existe controversia en este punto (Vergara, 1992).

Dentro de los componentes de la textura, el contenido de arcilla es el mas determinante que influye en el encalado, ya que un mayor contenido de ella en el suelo origina una mayor capacidad de intercambio catiónico y una mayor cantidad de iones que pueden ser adsorbidos por los sitios de intercambio y entre más sitios de intercambio haya interlaminarmente, será mayor el número de polímeros de aluminio que se acumulen entre las capas de los cristales de las arcillas de los suelos ácidos y consecuentemente una mayor cantidad de material alcalinizante para elevar el $\mathrm{pH}$ inicial del suelo a un nivel deseado. Por ello el suelo "El Progreso" necesito mayor cantidad de $\mathrm{CaCO}_{3}$ para elevar el $\mathrm{pH}$ ya que tiene un contenido mayor de arcilla y materia orgánica que el de "Las Animas".

La capacidad máxima de retención de agua fue medido para el suelo de cada sitio muestreado, debido a que su valor se ocupó para el cálculo del volumen de agua necesario $(60 \pm 5 \%)$ en la incubación del suelo con las dosis crecientes de cal, siendo para "Las Animas" de 222 mL Kg ${ }^{-1}$ de suelo y para "El Progreso" fue de $264 \mathrm{~mL} \mathrm{Kg}$ ${ }^{1}$ de suelo.

\section{CONCLUSIONES}

Los resultados de este trabajo muestran que los métodos Houba y Van Schouwenburg, Dunn y SMP-Simple permiten estimar RC, para los $\mathrm{pH}$ considerados, con mayor exactitud que los métodos de Keeney y Corey (1963), Adams y Evans (1962), Acidez hidrolítica o de Kappen modificado (1976), Acidez intercambiable (1970) y el de Morejon (1988).

SMP-Simple presenta la ventaja de que permite estimar los RC de manera rápida, a partir de una lectura de $\mathrm{pH}$ en una suspensión suelo-solución amortiguadora, lo que permite un análisis de rutina seguro e inmediato en el laboratorio.

Los suelos Acrisoles ferricos evaluados tiene un $\mathrm{pH}$ fuertemente acido, por consiguiente tienen una elevada acidez toxica y una pobreza en las bases de intercambio.

Las dosis crecientes de $\mathrm{CaCO}_{3}$ a los suelos de los sitios muestreados aumentaron el $\mathrm{pH}$, el calcio intercambiable, la capacidad de intercambio catiónica efectiva, el porcentaje de saturación de calcio y el de bases, así mismo, disminuyeron la acidez y aluminio intercambiables y el porcentaje de saturación de aluminio. Los 
cationes magnesio y potasio no fueron modificados.

El máximo rendimiento de materia seca adquirido en el experimento de invernadero para el cultivo del maíz (Zea mays) con la aplicación de $\mathrm{CaCO}_{3}$, se obtuvo para ambos sitios estudiados con la dosis de 6 ton $\mathrm{ha}^{-1}$ correspondiente al tratamiento No. 4 .

No existen diferencias significativas en las estimaciones del requerimiento de cal para los pH 5.0, 5.5, 6.0 y 6.5 en los obtenidos por la metodología patrón o referencia y las que usan soluciones amortiguadoras.

La acidez causada por el aluminio intercambiable es suficiente para estimar los requerimientos de cal en suelos ácidos para esta región, utilizando el factor 2 para la ecuación de cálculo.

\section{LITERATURA CITADA}

Adams, F., and C. E. Evans. 1962. A rapid method for measuring lime requeriment of red- yellow podsolic soils. Soil Sci. Soc. Am. Proc. 26. f https://doi.org/10.2136/sssaj1962.0361599 $5002600040015 \mathrm{x}$

Cajuste, L. J. 1977. Química de suelos con un enfoque agrícola. Colegio de Postgraduados, Chapingo, Mexico.

CSTPA. 1980. Handbook on reference methods for soil testing (Revised edition). Concil Soil Testing and plant analysis. Athens, Georgia. USA.

Cottenie, A. 1980. Los análisis de suelos como base para formular recomendaciones sobre fertilizantes. Boletin de suelos de la FAQ 38:2. FAQ. Roma, Italia.
Etchevers, B. J. D., G. Espinosa, y E. Riquelme. 1971. Manual de fertilidad y fertilizantes 2a edition. Universidad de Concepción, Facultad de Agronomía, Chilian, Chile.

FAO/UNESCO. 1988. Soil Map of the World, Revised Legend. World. Resources Report 60. FAO, Rome.

Fassbender, H. y E. Bornemisza.1987. Química de suelos con énfasis en suelos de América Latina, Instituto Interamericano de Cooperación para la Agricultura, MCA. San José, Costa Rica.

INEGI. 1993. Anuario estadístico del Estado de Veracruz. Coordenadas geográficas y altitud de las cabeceras municipales. Instituto Nacional de Estadística, Geografía e lnformática, Veracruz, México.

Keeney, D.R. and R. B. Corey. 1963. Factors affecting the lime requirements of Winsconsin Soils. Soil Sci. Soc. Am. Proc. 27:277-280

https://doi.org/10.2136/sssaj1963.0361599 5002700030019x

Morejon, A. L. E. 1987. Evaluación de diferentes métodos y criterios para determinar requerimientos de cal en suelos tabacaleros de la provincia de <pinar del Rio. Tesis de Doctorado. Ministerio de la Agricultura. Instituto de suelos. Ciudad Habana, Cuba.

Moreno, D. R. 1988. Clasificación del pH del suelo, contenido de sales y nutrimentos asimilables. INIA-SARH. México.

Rossignol, J.P. 1988. Morfología del área Xalapa-Coatepec. Mapa escala 1:75000 ORSTOM- INIREB. Veracruz, México.

Soto E., M., y M. Gómez C. 1993. Consideraciones climáticas de la Ciudad de Xalapa. Pp 81-98. In; López-Moreno 
Ismael R. (ed). Ecología urbana aplicada a la Ciudad de Xalapa. Instituto de Ecología. México.

Vergara Sánchez, M. A. 1992. Problemas nutrimentales y e! uso de fertilizantes en andosoles. Departamento de suelo. Universidad Autónoma de Chapingo. Chapingo, México.

Copyright (c) 2019 Doris G. Castillo Rocha, Ana C. Segura Castilla, Maria de J. Martinez Hernández, Liliana Lara Capistrán y Enrique Aguirre López

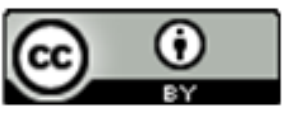

Este tex to está protegido por una licencia CreativeCommons 4.0

Usted es libre para Compartir —copiar y redistribuir el $\mathrm{m}$ aterial en cualquier medio o formato- $\mathrm{y}$ Adaptar el documento —remezclar, transformar y crear a partir del material- para cualquier propósito, incluso para fines com erciales, siempre que cumpla la condición de:

Atribución: Usted debe dar crédito a la obra original de manera adecuada, proporcionar un enlace a la licencia, e in dicar si se han realizado cambios. Puede hacerlo en cualquier forma razonable, pero no de forma tal que sugiera que tiene el apoyo del licenciante olo recibe por el uso que hace de la obra.

Resumendelicencia - Textocompletodelalicencia 